\title{
CONTRIBUTION A L'ÉTUDE DES ÉCHANGES ÉNERGÉTIQUES DU MOUTON
}

PAR

\author{
A. M. LEROY et S. Z. ZELTER
}

Laboratoire de Recherches de Zootechnie de l'Institut National Agronomique, Paris

\section{PLAN DU MEMOIRE}

I. - Exposé du problème.

II. - Méthodes utilisées au cours des recherches.

III. - Résultats expérimentaux.

IV. - Détermination de l'action dynamique spécifique des aliments ingérés en fonction de leur teneur en matière sèche.

V. - Conclusion : utilisation des résultats obtenus pour le calcul des rations destinées aux moutons.

\section{I. - EXPOSE DU PROBLEME}

Très peu d'expériences complètes de nétabolisme, avec détermination des ingesta et des excreta solides, liquides et gazeux ont été effectués sur des animanx de l'espèce ovine. En raison de leur faible poids, ces derniers se prêtent cependant fort bien aux essais en laboratoire, et nous avons utilisé cette facilité pour étudier par comparaison l'utilisation des fourrages par les agneaux ell cours de sevrage et par des adultes. Nous avons observé des sujets appartenant à la race Ile-de-lirance et à la race de Texel, comprenant des agneaux de 2 à 4 mois, des brebis en lactation et une brebis récemment séparée de son petit et ne donnant plus de lait.

\section{' II. - METHODES UTILISEES AU COURS DES RECHERCHES}

Au cours de ces recherches, les animaux ont été placés dans des cages métalliques suffisamment grandes pour leur permettre de se mouvoir aisément. Ces cages étaient munies dans leur partie inférieure d'un 
vaste réservoir en forme d'entonnoir, surmonté d'un jeu de grilles, permettant la séparation facile des fècès et de l'urine. Pour mesurer les échanges gazeux, ces cages pouvaient être recouvertes à volonté d'une enveloppe métallique rigide, munie de hublots, que des joints hydraulicutres permettaient de rendre étanche. Un jen de palans rendait à la fois aisée et rapide la manouvre de l'appareil. Ce dispositif, par son fonctionnement, ménageait à l'animal un volume d'air de I 400 litres, ce qui permettait la mesure précise des échanges gazeux, pendant des durées variant de 20 à 4.5 minutes, par la méthode de confinement. Des analyses de gaz, effectuées immédiatement avant et après chaque opération, nous faisaient connaitre la consommation d'oxygène ainsi que l'émission de gaz carbonique et de méthane pendant la durée de l'expérience. In répétant ces opérations aux diverses heures de plusieurs journées successives, il nous était permis de suivre l'évolution de la dépense d'énergie de nos animaux, par la connaissance que nous avions de leur consommation d'oxygène et du quotient respiratoire $\frac{\mathrm{CO}_{2}}{\mathrm{O}_{2}}$ correspondant. Nous avions ainsi la faculté d'observer la dépense énergétique de chaque sujet pendant ses repas, ce qui n'aurait pas été possible si nous avions utilisé des masques respiratoires, comme l'avaient fait auparavant d'autres expérimentateurs.

Des analyses nombreuses nous faisaient connaitre également la composition des aliments ingérés, et particulièrement leur teneur en azote et en carbone. Les excréments solides et liquides ont été également analysés avec soin. A plusieurs reprises, nous avons fait dans la cloche des prélèvements d'air d'une vingtaine de litres, qui nous ont servi pour le dosage du méthane, après combustion à la grille de ce dernier pour obtenir sa transformation en gaz carbonique.

Les quantités d'énergie brute correspondant aux ingesta, aux fècès et à la matière sèche de l'urine ont été évaluées après combustion dans une bombe calorimétrique de Ferry. I,es renseignements recueillis nous ont ainsi permis, pour des périodes d'observation de quatre à cinq jours, d'établir les bilans du carbone et de l'azote de nos animaux, et d'en déduire, par la méthode de calorimétrie indirecte correspondante, la quantité d'énergie dépensée quotidiennement par l'animal. Ceci nous a procuré la possibilité de vérifier l'exactitude des mesures de métabolisme directement déduites de la consommation d'oxygène, dont les données se trouvaient ainsi assez bien recoupées, en dépit de quelques petites erreurs inévitables en raison de la longue durée et de la technique de nos essais.

'Toutes ces observations ont été faites dans un local dont la température s'est maintenue constamment entre I5 et I $8^{\circ}$, ce qui excluait toute possibilité de l'intervention d'un mécanisme de régulation ther- 
mique capable, par le coût supplémentaire d'énergie qu'il aurait entraîné, d'apporter des perturbations dans nos mesures. Enfin, nous croyons utile de rappeler que nous avons toujours fait nos prélèvements de gaz dans la cloche après brassage de l'atmosphère au moyen d'un ventilateur, et que nous n'avons jamais oublié de tenir compte des corrections de température et de pression indispensables à l'exactitude des analyses de gaz.

\section{III. - RESULTATS EXPERIMENTAUX}

A titre d'exemple, nous indiquons ci-dessous, pour un agneat sevré âgé de 3 mois, la variation des échanges respiratoires en fonction de ces mêmes échanges mesurés de six à huit heures du matin, pendant le repos, sur un sujet à jeun, depuis la veille, à I7 heures. La quantité d'oxygène mesurée dans ces conditions a été prise conventionnellement égale à Ioo.

\section{TABLEAU I}

Variation de la consommation horaire d'oxygène d'un agneau récemment sevré, au cours d'une période de 24 heures.

\begin{tabular}{|c|c|c|c|c|c|c|}
\hline $\mathrm{de}_{\mathrm{à}} 6$ & $\begin{array}{l}\text { Pendant le } \\
\text { le repas du }\end{array}$ & \multicolumn{5}{|c|}{$\begin{array}{c}\text { Pendant le repas } \\
\text { du soir }\end{array}$} \\
\hline $8 \mathrm{~h}$ & matin $10 \mathrm{~h}$ & I I $\mathrm{h}$ & $I_{4} \mathrm{~h}$ & I $7 \mathrm{~h}$ & $19 \mathrm{~h}$ & $22 \mathrm{~h}$ \\
\hline - & $\cdots$ & 一 & - & 一 & - & - \\
\hline 100 & $13^{8}$ & 120 & 105 & $15 \mathrm{I}$ & I 24 & 120 \\
\hline
\end{tabular}

Au cours de cette expérience, la dépense horaire minimum, observée dans la matinée, était de 75 calories, alors que celle du métabolisme d'entretien correspondant, calculée d'après le poids du sujet - 3I $\mathrm{kg}$ était de 40,5 calories ( $\left.{ }^{1}\right)$.

Pour mieux étudier le phénomène de la hausse du métabolisme, qui se produit pendant le repas, nous avons distribué au même animal, la semaine suivante, des repas composés chacun de $75 \mathrm{~g}$ de mélange concentré et de $150 \mathrm{~g}$ de carottes, correspondant à $\mathrm{I} 5 \mathrm{O} \mathrm{g}$ de matière sèche. A partir de Io heures, les repas se sont succédés toutes les deux heures et demie, jusqu'au dernier, consommé à partir de $22 \mathrm{~h} 30$.

Les aliments ont été mangés dans l'appareil à confinement, et la durée de chacun de ces six repas a été limitée à 30 minutes. Comme les précédents, les résultats de la consommation horaire d'oxygène pour les différentes expériences ont été comparés au métabolisme minimum de la journée, mesuré à six heures du matin.

(1) Il s'agit ici, comme dans toute la suite de cet exposé, de grandes calories, c'est-ii-dire de calories-kilogranme.

Annales de Zootechnie. - I954 


\section{TABLEAU II}

Variation de la consommation horaire d'oxygène d'un agneau recevant toutes les deux heures $\mathrm{I} / 2$ des repas apportant chacun $150 \mathrm{~g}$ de matière sèche.

\begin{tabular}{|c|c|c|c|c|c|c|c|c|}
\hline à : $6 \mathrm{~h}$ & $\begin{array}{c}\text { Io } h \\
\text { I }^{\text {er repas }} \\
\end{array}$ & $\underset{\mathrm{I}^{\text {re }}}{\mathbf{I} \text { l }}$ & $\begin{array}{l}\text { h I } 5 \\
\text { inter. } \\
-\end{array}$ & $\begin{array}{l}\text { I } 2 \\
2^{\mathrm{e}}\end{array}$ & $\begin{array}{l}\text { h } 30 \\
\text { repás } \\
\end{array}$ & $\begin{array}{r}{ }^{1} 3 \mathrm{~h} 45 \\
2^{\mathrm{e}} \text { interv. }\end{array}$ & $\begin{array}{c}\text { I } 5 \mathrm{~h} \\
3^{\mathrm{e}} \text { repas } \\
-\end{array}$ & $\begin{array}{r}16 \mathrm{~h} \mathrm{I} 5 \\
3^{\mathrm{e}} \text { interv }\end{array}$ \\
\hline I00 & II9 & & 114 & & 134 & 119 & I +9 & 124 \\
\hline $\begin{array}{l}\text { I h } 30 \\
4^{\mathbf{e}} \text { repas } \\
-\end{array}$ & $4^{\mathrm{e} 8 \mathrm{~h}}$ & & $5^{2} \mathrm{r}$ & & & $\begin{array}{l}h \text { I } 5 \\
\text { interv. } \\
-\end{array}$ & $\begin{array}{l}22 \mathrm{~h} 30 \\
6^{\mathrm{e}} \text { repas }\end{array}$ & $6^{e} \stackrel{24 \mathrm{~h}}{\text { interv. }}$ \\
\hline
\end{tabular}

Des essais effectués selon le même principe ont été entrepris sur une brebis en lactation de la race de Texel, pesant $54 \mathrm{~kg}$, qui donnait par jour r.026 grammes de lait à 6,5 p. Ioo de matière grasse. Cette brebis a été alimentée pendant quatre jours au moyen de 4 repas quotidiens, séparés par des intervalles de 6 heures. La nourriture distribuée était formée d'un mélange d'orge, d'avoine, de son de blé, de tourteau de lin et de farine de luzerne. I a matière sèche ingérée en un repas était de 425 grammes.

La consommation horaire minimum d'oxygène, mesurée immédiatement avant l'un des repas, a été également prise ici comme terme de comparaison.

\section{Tableau III}

Variation de la consommation horaire d'oxygène d'une brebis Texel, recevant toutes les six heures des repas égaux apportant chacun $42 j$ grammes de matière sèche.

\begin{tabular}{|c|c|c|c|c|c|c|}
\hline $\begin{array}{l}6 \mathrm{~h} \text { après le dernier repas } \\
\text { Inmédiatement avant le } \\
\text { repas suivant }\end{array}$ & $\begin{array}{l}\text { Pendant } \\
\text { le } \\
\text { repas }\end{array}$ & $\begin{array}{l}\text { Après } \\
2 \mathrm{~h}\end{array}$ & $\begin{array}{c}\text { Apres } \\
3 \mathrm{~h}\end{array}$ & $\begin{array}{c}\text { Apres } \\
+h\end{array}$ & $\begin{array}{c}\text { Apris } \\
5 \mathrm{~h}\end{array}$ & $\begin{array}{l}\text { Moyenne } \\
\text { pour la } \\
\text { journce }\end{array}$ \\
\hline- & - & 一 & - & - & - & - \\
\hline 100 & 155 & I 22 & 11.3 & 107 & 104 & I I 7 \\
\hline
\end{tabular}

En multipliant les prises d'essai, pour chaque animal, aux divers instants de la journée et de la nuit, il nous a été possible, au cours d'expériences durant chacune plusieurs jours, de construire par points la courbe des échanges gazeux de nos divers sujets, et d'en déduire aisément leur consommation moyenne horaire d'oxygène ainsi que le quotient respiratoire correspondant.

A titre d'exemple, nous reproduisons ci-dessous intégralement les données de deux expériences, dont l'une se rapporte à un agneau non sevré, et l'autre à une brebis. Tous nos essais, au nombre de II, ont donné lieu à l'établissement de tableaux établis d'après ces modèles, que nous nous excusons de ne pouvoir reproduire tous en détail. 
TABLEAU IV

Résultats d'une expérience effectuée sur un agneau non sevré :

Poids moyen du sujet ......... I8,3 kg Age........ 81 jours

Composition de l'aliment concentré utilisé

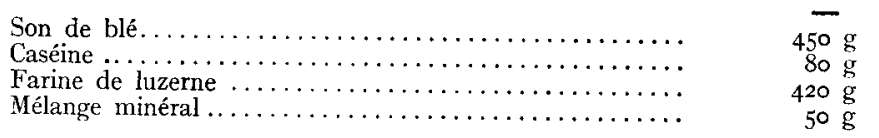

I. - Quantité et composition des aliments ingérés par jour :

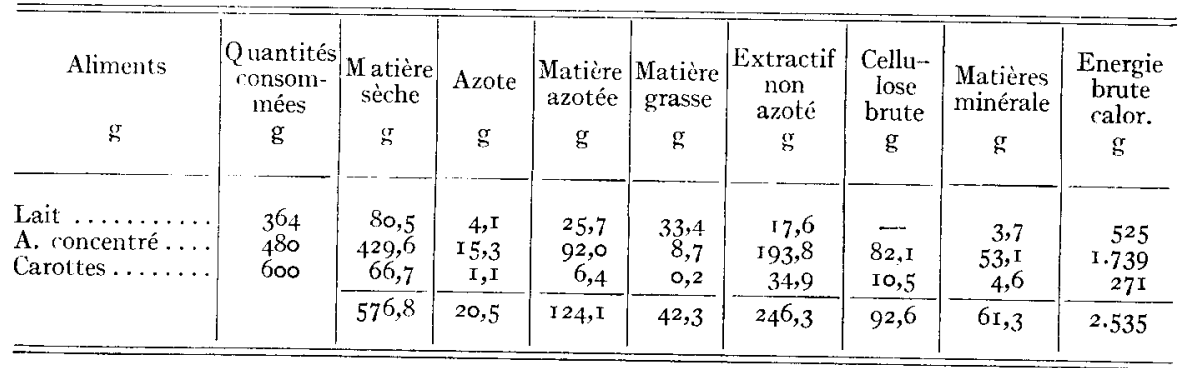

II. - Quantité et composition des excréments solides émis par jour :

\begin{tabular}{|c|c|c|c|c|c|c|c|}
\hline $\begin{array}{l}\text { Matière } \\
\text { secche }\end{array}$ & Azote & $\begin{array}{l}\text { Matière } \\
\text { azotée }\end{array}$ & $\begin{array}{l}\text { Matière } \\
\text { grasse }\end{array}$ & $\begin{array}{c}\text { Extractif } \\
\text { non azoté }\end{array}$ & $\begin{array}{c}\text { Cellulose } \\
\text { brute }\end{array}$ & $\begin{array}{l}\text { Matière } \\
\text { minérale }\end{array}$ & $\begin{array}{c}\text { Energie } \\
\text { brute } \\
\text { calor. }\end{array}$ \\
\hline$\underline{g}$ & $\underline{\mathrm{g}}$ & $\underline{s}$ & $g$ & g & $\mathrm{g}$ & $\mathrm{g}$ & $\mathrm{g}$ \\
\hline IOI,O & 3,1 & I9,2 & $\mathrm{I}, 8$ & 39,0 & 25,7 & $\sqrt{15,2}$ & $4 \overline{62}$ \\
\hline
\end{tabular}

III. - Quantité et composition de l'urine émise par jour :

$\begin{array}{ccccc}\text { Matière sèche } & \text { Azote } & \text { Matière organique } & \text { Matière minérale } & \begin{array}{c}\text { Energie brute } \\ \text { calories }\end{array} \\ \overline{34.7} & \overline{9,5} & \overline{26,0} & \overline{8,7} & \overline{70}\end{array}$

IV. - Balance de l'Azote :

$\begin{array}{cccc}\text { Azote ingéré } & \text { Azote fécal } & \text { Azote de l'urine } & \text { Azote retenu } \\ 20,5 & \frac{-}{3, \mathrm{r}} & \overline{9,5} & -\end{array}$

V. - Balance du Carbone:

$\begin{array}{cccccc}\begin{array}{c}\text { Carbone } \\ \text { ingéré }\end{array} & \begin{array}{c}\text { Carbone } \\ \text { fécal }\end{array} & \begin{array}{c}\text { Carbone } \\ \text { de l'urine }\end{array} & \begin{array}{c}\text { Carbone } \\ \text { du méthane }\end{array} & \begin{array}{c}\text { Carbone } \\ \text { dul } \mathrm{CO}_{\mathbf{2}}\end{array} & \begin{array}{c}\text { Carbone } \\ \text { retenu }\end{array} \\ \frac{\mathbf{2 4 5 , 5}}{\mathrm{g}} & \frac{\mathrm{g}}{\mathrm{g}} & \frac{\mathrm{g}}{\mathrm{g}, 3} & \frac{\mathrm{g}}{2,7} & \frac{\mathrm{g}}{\mathrm{g}} & \frac{\mathrm{g}}{52,0}\end{array}$

VI. - Balance de l'énergie :

\begin{tabular}{|c|c|c|c|c|c|}
\hline 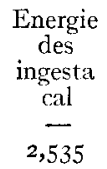 & $\begin{array}{c}\begin{array}{c}\text { Energie } \\
\text { des }\end{array} \\
\text { fecès } \\
\text { cal } \\
\overline{4 \delta_{2}}\end{array}$ & $\begin{array}{c}\text { Energie } \\
\text { de } \\
\text { l'urine } \\
\text { cal } \\
\frac{70}{70}\end{array}$ & $\begin{array}{l}\text { Energie } \\
\text { du } \\
\text { méthane } \\
\text { cal } \\
\overline{48}\end{array}$ & $\begin{array}{c}\text { Energie } \\
\text { retenue } \\
\frac{\text { cal }}{573}\end{array}$ & $\begin{array}{l}\text { Energie dépensée sous } \\
\text { forme de chaleur } \\
\text { (mesurée) } \\
\text { cal } \\
\overline{\mathrm{I}, 382}\end{array}$ \\
\hline
\end{tabular}




\section{VII. - Analyse des Composants organiques des tissus de croissance :}

a) Matière azotée :

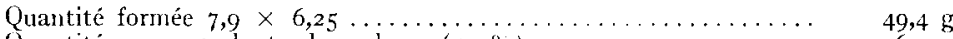

Quantité correspondante de carbone $\left(53{ }_{i}\right) \ldots \ldots \ldots \ldots \ldots \ldots \ldots, 26,2$ g

Fnergie correspondante $(5,55$ cal. par $g) \ldots \ldots \ldots \ldots \ldots \ldots \ldots \ldots \ldots \ldots \ldots \ldots$. 74 calories

b) Matière grasse :

Quantité correspondante de carbone $52-26,2 \ldots \ldots \ldots \ldots \ldots \ldots 25,8 \mathrm{~g}$

Quantité formée $\frac{25,8 \times 1000}{765} \ldots \ldots \ldots \ldots \ldots \ldots \ldots \ldots \ldots \ldots \ldots \ldots \ldots \ldots \ldots \ldots, 73,7 \mathrm{~g}$

Énergie correspondante $(9,2$ cal. par g) .............. \$10 calories

Quantité d'énergie calculée d'après les bilans d'àote et de arbont ........ 584 calories

Quantité d'énergie calculée d'après le bilan d'énergie $\ldots \ldots \ldots \ldots \ldots \ldots \ldots \ldots \ldots . \ldots \ldots$ calories

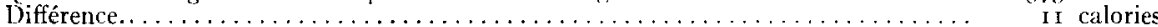

\section{TABLFAU V}

\section{Résultats d'une expérience sur brebis après sevrage de son agneau}

\begin{tabular}{|c|c|c|c|c|c|c|c|c|c|}
\hline$\Lambda$ limeness & $\begin{array}{l}\text { Quantités } \\
\text { consom- } \\
\text { mées } \\
\mathrm{g}\end{array}$ & $\begin{array}{l}\mathrm{Ma}- \\
\text { tières } \\
\text { sèche } \\
\mathrm{g}\end{array}$ & $\begin{array}{c}\text { Azote } \\
g\end{array}$ & $\begin{array}{l}\text { Ma- } \\
\text { tière } \\
\text { azotée } \\
\mathrm{g}\end{array}$ & $\begin{array}{l}\text { Ma- } \\
\text { tière } \\
\text { grasse } \\
\mathrm{g}\end{array}$ & $\begin{array}{l}\text { Extractif } \\
\text { non } \\
\text { azote } \\
g\end{array}$ & $\begin{array}{l}\text { Cellu- } \\
\text { lose } \\
\text { brute } \\
g\end{array}$ & $\begin{array}{l}\text { Matières } \\
\text { miné- } \\
\text { rales } \\
g\end{array}$ & $\begin{array}{c}\text { Énergie } \\
\text { brute } \\
\text { calor } \\
\text { g }\end{array}$ \\
\hline - & - & - & 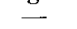 & - & $\ldots$ & 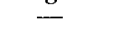 & - & $\approx$ & 6 \\
\hline Farine de luzeme. & 125 & II 3,0 & 3,5 & $2 \mathrm{I}, 2$ & $\mathrm{x}, 2$ & 53,8 & 29,9 & 6,8 & 487 \\
\hline Orge $\ldots \ldots \ldots \ldots$ & 300 & 258,5 & 4,9 & 20,4 & 5,2 & 193,5 & 22,7 & 7,7 & 1080 \\
\hline Avoine .......... & 250 & 219,8 & 5,1 & $30, \mathrm{I}$ & 10,3 & I 33,6 & 37,6 & 8,1 & 956 \\
\hline Betteraves....... & 4000 & $5^{82,0}$ & 9,4 & $5^{6,6}$ & 1,2 & 459,8 & 31,7 & 32,6 & 2217 \\
\hline Carottes. & 1000 & Jo7,8 & 1,8 & I I , O & 0,4 & $7 \mathrm{~T}, 7$ & 17,0 & 7,6 & $43^{8}$ \\
\hline Foin & 500 & 449,4 & 8,6 & 50,7 & I I,4 & 209,5 & 26,4 & 51,6 & I 843 \\
\hline \multirow{3}{*}{ - à déduire, refus } & & 730 & 33,3 & 199,0 & 29,7 & $1,21,0$ & 265,3 & 114,4 & 7021 \\
\hline & & & 2,8 & 16,5 & 3,1 & $95+3^{3}$ & $5^{6,6}$ & 14,6 & 762 \\
\hline & & $154,3.7$ & 30,5 & 182.5 & 26,6 & 1026.1 & 208,7 & 99,8 & 6259 \\
\hline
\end{tabular}

II. - Quantité et composition des excréments solides :

$\begin{aligned} & \text { Matière } \\ & \text { zéche }\end{aligned}$
$\begin{aligned} & \text { Azote } \\ & \frac{1}{402,3}\end{aligned}$

\section{III. - Quantité et composition de l'urine :}

\begin{tabular}{|c|c|c|c|c|}
\hline $\begin{array}{l}\text { Maticre } \\
\text { siche }\end{array}$ & Azote & $\begin{array}{l}\text { Maticre } \\
\text { organique }\end{array}$ & $\begin{array}{l}\text { Matiere } \\
\text { minúrale }\end{array}$ & $\begin{array}{c}\text { Energie } \\
\text { brute }\end{array}$ \\
\hline$g$ & $g$ & $\mathrm{~g}$ & $g$ & ralories \\
\hline - & - & 一 & - & - \\
\hline$s+5$ & 10,0 & 35,0 & -4), 5 & 171 \\
\hline
\end{tabular}

\section{IV. - Balance de l'Azote :}

\begin{tabular}{|c|c|c|c|}
\hline Azote ingere & Azote fecal & Azole de l'urine & Azole du bilan \\
\hline$\underline{-}$ & $\because$ & $\underline{I}$ & $\ddot{s}$ \\
\hline 30,5 & $\overline{\mathrm{Ii}, 4}$ & 10,9 & 8,2 \\
\hline
\end{tabular}




\section{V. - Balance du Carbone:}

\begin{tabular}{|c|c|c|c|c|c|}
\hline $\begin{array}{l}\text { Carbone } \\
\text { ingéré }\end{array}$ & $\begin{array}{c}\text { Carbone } \\
\text { fécal }\end{array}$ & $\begin{array}{l}\text { Carbone } \\
\text { de l'urine }\end{array}$ & $\begin{array}{l}\text { Carbone } \\
\text { du méthane }\end{array}$ & $\begin{array}{l}\text { Carbone } \\
\text { du } \mathrm{CO}_{2}\end{array}$ & $\begin{array}{l}\text { Carbone } \\
\text { du bilan }\end{array}$ \\
\hline $\mathrm{g}$ & g & $\mathrm{g}$ & $\mathrm{g}$ & $\mathrm{g}$ & $\mathrm{g}$ \\
\hline$\overline{G_{11}}$ & $\overline{I G I, O}$ & $1 \overline{8,5}$ & 23,4 & $33 \mathrm{I}, 5$ & 76,6 \\
\hline
\end{tabular}

VI. - Balance de l'Energie:

\begin{tabular}{|c|c|c|c|c|c|}
\hline $\begin{array}{c}\text { Energie } \\
\text { des ingesta } \\
\text { cal } \\
\end{array}$ & $\begin{array}{c}\text { Energie } \\
\text { des féiés } \\
\text { cal }\end{array}$ & $\begin{array}{l}\text { Energie } \\
\text { de l'urine } \\
\text { cal } \\
\end{array}$ & $\begin{array}{c}\text { Energie } \\
\text { du méthane } \\
\text { cal } \\
-\end{array}$ & $\begin{array}{l}\text { Energie du } \\
\text { métabolisme } \\
\text { cal } \\
-\end{array}$ & $\begin{array}{c}\text { Energie } \\
\text { du bilan } \\
\text { cal } \\
\end{array}$ \\
\hline 6259 & 1605 & 175 & +18 & 3006 & 1059 \\
\hline
\end{tabular}

VII. - Analyse des composants organiques des tissus de croissance.

a) Matière azotée :

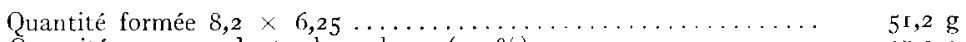

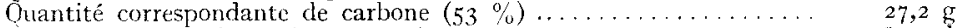

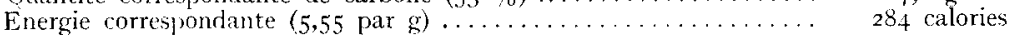

b) Matière grasse :

Quantité correspondante de carhone $i 6,6 \ldots 27,2 \ldots \ldots \ldots \ldots \ldots . \quad 49,4 \mathrm{~g}$

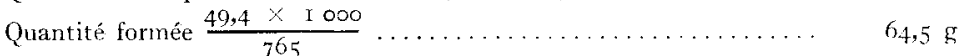

Énerrie correspondante $(9,2$ cal. par $g) \ldots \ldots \ldots \ldots \ldots \ldots \ldots \ldots \ldots$ calories.

Quantité d'énergie calculée d'après les bilans d'azote et de carbone ........ 877 calories:

Guantité d'énerogie calculée d'après le bilan de l'énergie ............... I 059 _...

Différence. . . . . . . . .

Voici, maintenant, les résultats des I I expériences entreprises, dont 8 sur agneaux sevrés et non sevrés, et 3 sur brebis en lactation et tarie. Pour chacune de ces expériences, nous avons indiqué les bilans de l'énergie, de l'azote et des matières azotées, du carbone et des matières grasses. Les données ainsi recueillies nous ont permis, en outre, de calculer l'énergie des gains ou pertes organiques de nos animaux par les deux méthodes différentes de la calorimétrie indirecte.

\section{TABLEAU VI}

A. Expériences sur agneaux

I. - Bilans de l'énergie - Agneaux non-sevrés :

\begin{tabular}{|c|c|c|c|c|c|c|c|c|c|c|}
\hline & $\begin{array}{l}\text { No de } \\
\text { l'essai }\end{array}$ & $\begin{array}{l}\text { Poids } \\
\text { dus } \\
\text { sujet } \\
\text { kor }\end{array}$ & $\begin{array}{l}\text { Mat. } \\
\text { sèche } \\
\text { incurére }\end{array}$ & $\begin{array}{l}\text { Ener- } \\
\text { gie } \\
\text { des } \\
\text { ingrestal } \\
\text { cal. }\end{array}$ & $\begin{array}{l}\text { Ener- } \\
\text { gic } \\
\text { des } \\
\text { ferès } \\
\text { cal. }\end{array}$ & $\begin{array}{l}\text { Ener- } \\
\text { grie } \\
\text { de } \\
\text { l'urine } \\
\text { cal }\end{array}$ & $\begin{array}{l}\text { Ener- } \\
\text { gie du } \\
\text { mé- } \\
\text { thane } \\
\text { cal. }\end{array}$ & $\begin{array}{l}\text { Éner- } \\
\text { gie } \\
\text { métabo- } \\
\text { lisable } \\
\text { cal. }\end{array}$ & $\begin{array}{c}\text { Éner- } \\
\text { gie } \\
\text { perdue } \\
\text { (mesurée) } \\
\text { cal. }\end{array}$ & $\begin{array}{l}\text { Éner- } \\
\text { gie } \\
\text { du } \\
\text { bilan } \\
\text { cal. }\end{array}$ \\
\hline & 一 & - & $\underline{5}$ & - & $\ldots$ & $\ldots$ & - & $\ldots$ & - & - \\
\hline$[-307$ & $\ldots \ldots \ldots \ldots$ & 18,3 & 576,8 & 2.535 & 462 & 70 & 48 & I 953 & I 382 & 573 \\
\hline $2-31+$ & $\ldots \ldots \ldots \ldots$ & 19,2 & 525,4 & 2816 & 305 & 79 & 33 & 2399 & I $22 \mathrm{I}$ & I 178 \\
\hline $3-314$ & $\ldots \ldots \ldots$ & 22,6 & $54^{6,2}$ & 2609 & 612 & 104 & 34 & [ 859 & I 334 & $5^{25}$ \\
\hline $4=314$ & $\ldots \ldots$ & 25,0 & 406,7 & 2070 & 463 & $6 I$ & $\ldots$ & I 555 & I 345 & 210 \\
\hline $5^{-} 3^{1}-4$ & & $28, \mathbf{I}$ & 836,0 & 3755 & I 046 & 105 & 59 & 2545 & 1909 & 636 \\
\hline $6-31+$ & & 30,7 & 728,0 & 3012 & I 005 & 59 & 57 & $\mathrm{I} 89 \mathrm{I}$ & $182 \mathrm{I}$ & 70 \\
\hline $7-314$ & $\ldots \ldots$ & 31,0 & 696,0 & 2882 & 670 & II 9 & 63 & 2030 & 1505 & $5^{2} 5$ \\
\hline $8-3^{T}+1$ & $\ldots \ldots \ldots \ldots$ & 32,8 & 817,0 & $355^{6}$ & 954 & I 18 & 72 & 2412 & 1892 & 20 \\
\hline
\end{tabular}




\section{II. - Bilans de l'Azote et des matières azotées :}

\begin{tabular}{|c|c|c|c|c|c|c|c|c|}
\hline & & & $A g$ & $u x \quad n$ & sevr & & & \\
\hline & $\begin{array}{l}N^{\circ} \text { du } \\
\text { sujet }\end{array}$ & $\begin{array}{l}\text { Azote } \\
\text { ingéré } \\
g\end{array}$ & $\begin{array}{c}\text { Azote } \\
\text { fécal } \\
g\end{array}$ & $\begin{array}{c}\text { Azote } \\
\text { urinaire } \\
g\end{array}$ & $\begin{array}{c}\text { Azote } \\
\text { du } \\
\text { bilan } \\
\text { g }\end{array}$ & $\begin{array}{c}\text { Matières } \\
\text { azotées du } \\
\text { bilan } \\
\sigma\end{array}$ & $\begin{array}{c}\text { Carbone } \\
\text { corres- } \\
\text { pondant } \\
g\end{array}$ & $\begin{array}{c}\text { Énergie } \\
\text { corres- } \\
\text { pondante } \\
\text { cal. }\end{array}$ \\
\hline & - & $\underline{\mathrm{g}}$ & $\underline{\mathrm{g}}$ & $\underline{3}$ & $\underline{\mathrm{g}}$ & $\underline{8}$ & s & Cal. \\
\hline$I-307$. & $\ldots \ldots \ldots \ldots$ & 20,5 & $3, \mathrm{I}$ & 9,5 & 7,9 & 49,4 & 26,2 & 277 \\
\hline $2 \cdot 3^{\mathrm{I}} 4$ & $\ldots \ldots \ldots \ldots$ & 20,8 & 3,1 & 8,6 & $9, \mathrm{I}$ & 56,9 & 30,1 & 316 \\
\hline $3 \cdot 314$ & $\ldots \ldots \ldots \ldots$ & 21,6 & 4,2 & I 2,9 & 4,5 & 28,1 & I 4,9 & I 56 \\
\hline $4-3$ I 4 & $\ldots \ldots \ldots \ldots$ & I 5,7 & 4,2 & 6.4 & $5, \mathrm{I}$ & 31,9 & 16,9 & I 77 \\
\hline $5-3$ I 4 & $\ldots \ldots \ldots$ & $3 \mathrm{I}, \mathrm{O}$ & 6,2 & 13,7 & I I, I & 69,4 & 36,8 & $3^{8} 5$ \\
\hline & & & & $a u x$ & $e ́ s$ & & & \\
\hline$-3 I_{4}$ & $\ldots \ldots \ldots$ & 25,4 & 5,5 & 13,2 & 6,7 & $4 I, 9$ & 22,2 & 232 \\
\hline $7-3 I_{4}$ & $\ldots$ & 23,4 & 4,8 & I $4, \mathrm{I}$ & 4,5 & $28, \mathrm{I}$ & 14,9 & I 56 \\
\hline $8-3 \mathrm{I} 4$ & & 29,4 & 6,5 & $\mathrm{I} 2, \mathrm{I}$ & 10,8 & 67,5 & 35,8 & 375 \\
\hline
\end{tabular}

III. - Bilans du carbone et des matières grasses :

Agneaux non-sevrés

\begin{tabular}{|c|c|c|c|c|c|c|c|c|c|}
\hline $\begin{array}{l}\text { No du } \\
\text { sujet }\end{array}$ & $\begin{array}{c}\text { Carbone } \\
\text { ingéré } \\
\mathrm{g}\end{array}$ & $\begin{array}{c}\text { Car- } \\
\text { bone } \\
\text { fécal } \\
\mathrm{g}\end{array}$ & $\begin{array}{l}\text { Car- } \\
\text { bone } \\
\text { urinaire } \\
\mathrm{g}\end{array}$ & $\begin{array}{c}\text { Car- } \\
\text { bone } \\
\text { du } \\
\mathrm{CH}_{4} \\
\mathrm{~g}\end{array}$ & $\begin{array}{c}\text { Car- } \\
\text { bone } \\
\mathrm{du} \\
\mathrm{CO}_{2} \\
\mathrm{~g}\end{array}$ & $\begin{array}{c}\text { Car- } \\
\text { bone } \\
\text { du } \\
\text { bilan } \\
\text { g }\end{array}$ & $\begin{array}{c}\text { Carbone } \\
\text { des } \\
\text { mat. } \\
\text { grasses } \\
\text { g }\end{array}$ & $\begin{array}{l}\text { Mat. } \\
\text { gr. } \\
\text { du } \\
\text { bilan } \\
\text { g }\end{array}$ & $\begin{array}{l}\text { Énergi } \\
\text { corres } \\
\text { pond. } \\
\text { cal }\end{array}$ \\
\hline$-307 \ldots$ & 245,5 & $\overline{35,3}$ & $\mathrm{I} \overline{6,0}$ & 2,7 & I 39,5 & 52,0 & 25,8 & 33,7 & 10 \\
\hline $2 \cdot 3 \mathrm{I} 4 \ldots$ & 265,0 & 25,2 & r4,5 & I, 9 & $\mathrm{I} \mathbf{2 2} 26$ & 100,7 & 70,5 & 92,2 & \\
\hline $3-3 \times 4 \ldots$ & 253,2 & 49,0 & 22,0 & 1,9 & I 34,9 & 45,4 & 30,5 & 39,8 & \\
\hline 35 & 209,5 & 3 & 10,9 & & I 33,8 & 25,7 & 8,8 & I 1,5 & I06 \\
\hline$-3^{14}$ & $37 \mathrm{~T}, 5$ & 90,2 & 23.0 & 3,8 & $\begin{array}{l}197,0 \\
1070\end{array}$ & 57,5 & 20,7 & $27, \mathrm{I}$ & 249 \\
\hline \multicolumn{10}{|c|}{ Agneaux sevrés } \\
\hline 314 & & 86,7 & 22,5 & 3,2 & I $9 \mathrm{I}, 7$ & 3,0 & $-19,2$ & $-25,0$ & -230 \\
\hline & & 68,8 & 24,0 & 3,5 & I 56,9 & 47,0 & 32 & 42,0 & \\
\hline 314 & $3^{67}, 0$ & 88,5 & 20,5 & 4,0 & 208,2 & 45,8 & 10,0 & $\mathrm{I} 3, \mathrm{~T}$ & $\mathrm{~T} 2$ \\
\hline
\end{tabular}

IV. - Comparaison des résultats obtenus par les deux modes de calcul.

A gneaux non-sevrés

\begin{tabular}{|c|c|c|c|c|c|}
\hline No du sujet & $\begin{array}{l}\text { Énergie } \\
\text { des mat. } \\
\text { azotées } \\
\text { cal. }\end{array}$ & $\begin{array}{l}\text { Energie } \\
\text { des mat. } \\
\text { grasses } \\
\text { cal. }\end{array}$ & $\begin{array}{c}\text { Gains ou } \\
\text { emprunts } \\
\text { d'énergie } \\
\text { (2 } 2^{\mathrm{e}} \text { méthode) } \\
\text { cal. }\end{array}$ & $\begin{array}{l}\text { Bilan } \\
\text { par } \\
\text { I re } \\
\text { méthode } \\
\text { cal. }\end{array}$ & $\begin{array}{c}\text { Différence entre } \\
\text { les résultats } \\
\text { des deux } \\
\text { méthodes } \\
\text { cal. }\end{array}$ \\
\hline - & - & 一 & - & - & - \\
\hline$-307 \ldots \ldots \ldots \ldots \ldots$ & 277 & $3^{10}$ & 587 & 573 & $-r_{4}$ \\
\hline $2 \cdot 314 \ldots \ldots \ldots \ldots \ldots$ & 316 & 848 & I I64 & I 178 & $+\mathrm{I}_{4}$ \\
\hline $3-3 \mathbf{I} 4 \ldots \ldots \ldots \ldots \ldots$ & $I_{5} 6$ & 366 & $5^{22}$ & 525 & +3 \\
\hline $4-3 \mathbf{I} 4 \ldots \ldots \ldots \ldots \ldots$ & I 77 & 106 & 283 & 210 & -73 \\
\hline $5-314 \ldots \ldots \ldots \ldots \ldots$ & 385 & 249 & 634 & 636 & +2 \\
\hline
\end{tabular}

\section{Agneaux sevrés}

\begin{tabular}{|c|c|c|c|c|c|}
\hline $6-324 \ldots \ldots \ldots$ & $\mathrm{r} 32$ & 230 & 2 & 70 & +68 \\
\hline $7-3$ เ $4 \ldots \ldots \ldots$ & 156 & 386 & 542 & 525 & -17 \\
\hline$-3 I 4 \ldots$ & 375 & 321 & 496 & 520 & +24 \\
\hline
\end{tabular}




\section{B. - Expériences sur brebis}

\section{I. - Brebis ayant sevré son agneau - Bilans de l'énergie}

\begin{tabular}{|c|c|c|c|c|c|c|c|c|c|}
\hline & & & & & & Ener- & & $\begin{array}{l}\text { Énergie } \\
\text { perdue }\end{array}$ & \\
\hline No du sujet & $\begin{array}{c}\text { Poids } \\
\text { du } \\
\text { sujet } \\
\mathrm{kg}\end{array}$ & $\begin{array}{c}\text { Mat. } \\
\text { sèche } \\
\text { ingérée } \\
\mathrm{g}\end{array}$ & $\begin{array}{c}\text { Énergie } \\
\text { des } \\
\text { ingesta } \\
\text { ('al. }\end{array}$ & $\begin{array}{c}\text { Energie } \\
\text { des } \\
\text { fecès } \\
\text { cal. }\end{array}$ & $\begin{array}{c}\text { Energie } \\
\text { de } \\
\text { l'urine } \\
\text { cal. }\end{array}$ & $\begin{array}{l}\text { gie du } \\
\text { mé- } \\
\text { thane } \\
\text { cal. }\end{array}$ & $\begin{array}{l}\text { Énergie } \\
\text { métabo- } \\
\text { lisable } \\
\text { cal. }\end{array}$ & $\begin{array}{l}\text { par le } \\
\text { métabol. } \\
\text { (mesur.) } \\
\text { cal. }\end{array}$ & $\begin{array}{c}\text { Energie } \\
\text { du } \\
\text { bilan } \\
\text { cal. }\end{array}$ \\
\hline 一 & - & - & - & - & 一 & - & - & $\rightarrow$ & - \\
\hline & 69,2 & I 5.44 & 6259 & I 605 & I 7 I & 418 & 4065 & 3006 & I 059 \\
\hline
\end{tabular}

Brebis en lactation :

\begin{tabular}{|c|c|c|c|c|c|c|c|c|c|c|}
\hline $\begin{array}{l}\text { No du } \\
\text { sirjet }\end{array}$ & $\begin{array}{c}\text { Poids } \\
\text { du } \\
\text { sujet } \\
\text { kg }\end{array}$ & $\begin{array}{l}\text { Mat. } \\
\text { sèche } \\
\text { ingérée } \\
g\end{array}$ & $\begin{array}{c}\text { Énergie } \\
\text { des } \\
\text { ingesta } \\
\text { cal. }\end{array}$ & $\begin{array}{c}\text { Énergie } \\
\text { des } \\
\text { fècès } \\
\text { cal. }\end{array}$ & $\begin{array}{c}\text { Énergie } \\
\text { de } \\
\text { l'urine } \\
\text { cal. }\end{array}$ & $\begin{array}{c}\text { Êner- } \\
\text { gie } \\
\text { dù } \\
\text { mé- } \\
\text { thane } \\
\text { cal. }\end{array}$ & $\begin{array}{c}\text { Énergie } \\
\text { métabo- } \\
\text { lisable } \\
\text { cal. }\end{array}$ & $\begin{array}{c}\text { Énergie } \\
\text { du } \\
\text { lait } \\
\text { cal. }\end{array}$ & $\begin{array}{c}\text { Énergie } \\
\text { perdue } \\
\text { par le } \\
\text { métabol. } \\
\text { (mesurée) } \\
\text { cal. }\end{array}$ & $\begin{array}{c}\text { Énergie } \\
\text { du } \\
\text { bilan } \\
\text { cal. }\end{array}$ \\
\hline - & - & - & - & - & - & - & - & - & - & - \\
\hline & 64,2 & & 89 & & 24 & $5^{8 \mathrm{I}}$ & 5226 & & & I 75 \\
\hline & 53,8 & 1702 & 7226 & 2733 & 309 & I 29 & 4055 & I 064 & 2897 & 94 \\
\hline
\end{tabular}

\section{II. - Bilans de l'azote et des matières azotées :}

Brebis ayant seuré son agneau :

\begin{tabular}{|c|c|c|c|c|c|c|c|}
\hline$N^{\circ} \mathrm{du}$ sujet & $\begin{array}{l}\text { Azote } \\
\text { ingéré } \\
g\end{array}$ & $\begin{array}{c}\text { Azote } \\
\text { fécal } \\
g\end{array}$ & $\begin{array}{c}\text { Azote } \\
\text { urinaire } \\
\mathrm{g}\end{array}$ & $\begin{array}{l}\text { Azote } \\
\text { du } \\
\text { bilan } \\
g\end{array}$ & $\begin{array}{c}\text { Matières } \\
\text { azotées } \\
\text { du bilan } \\
\mathrm{g}\end{array}$ & $\begin{array}{c}\text { Carbone } \\
\text { corres- } \\
\text { pondant } \\
g\end{array}$ & $\begin{array}{l}\text { Energie } \\
\text { correspon- } \\
\text { dante } \\
\text { cal. }\end{array}$ \\
\hline 一 & s & $\stackrel{5}{-}$ & 1 & $\frac{6}{2}$ & s & $\underline{8}$ & - \\
\hline $1-307 \ldots \ldots \ldots \ldots$ & 30,5 & I I, 4 & I0,9 & 8,2 & $5^{1,2}$ & $27, x$ & 284 \\
\hline
\end{tabular}

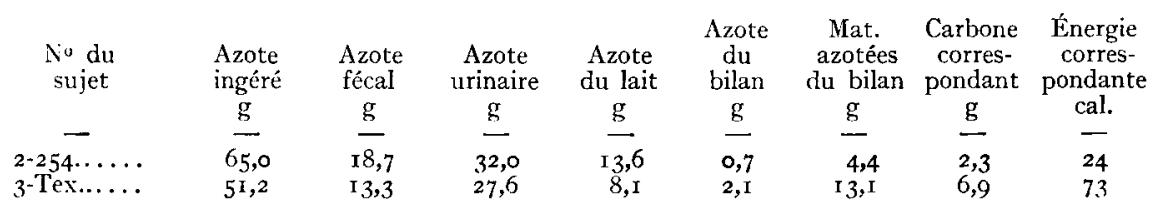

\section{III. - Bilans du carbone et des matières grasses}

\section{Brebis ayant sevré son agneau}

\begin{tabular}{|c|c|c|c|c|c|c|c|c|c|}
\hline $\begin{array}{l}\text { No du } \\
\text { sujet }\end{array}$ & $\begin{array}{c}\text { Carbone } \\
\text { ingéré } \\
\mathrm{g}\end{array}$ & $\begin{array}{l}\text { Carbone } \\
\text { fécal } \\
\text { g }\end{array}$ & $\begin{array}{c}\text { Carbone } \\
\text { urinaire } \\
\mathrm{g}\end{array}$ & $\begin{array}{c}\text { Carbone } \\
\text { du } \\
\mathrm{CH}_{4} \\
\mathrm{~g}\end{array}$ & $\begin{array}{c}\text { Carbone } \\
\text { du } \\
\mathrm{CO}_{2} \\
\mathrm{~g}\end{array}$ & $\begin{array}{c}\text { Carbone } \\
\text { du } \\
\text { bilan } \\
\mathrm{g}\end{array}$ & $\begin{array}{c}\text { Carbone } \\
\text { des mat. } \\
\text { grasses } \\
\mathrm{g}\end{array}$ & $\begin{array}{l}\text { Mat. } \\
\text { gr. du } \\
\text { bilan } \\
\text { g }\end{array}$ & $\begin{array}{l}\text { Énergie } \\
\text { corres- } \\
\text { pondante } \\
\text { cal. }\end{array}$ \\
\hline$\cdots$ & 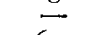 & & & 음 & 음 & & - & & - \\
\hline 307 & $61 \mathrm{I}$ & I6 I & I 8,5 & 23,4 & 331,5 & 76,6 & 49,5 & 64,8 & 596 \\
\hline
\end{tabular}

\section{Brebis en lactation}

\begin{tabular}{|c|c|c|c|c|c|c|c|c|c|c|}
\hline $\begin{array}{l}\text { No du } \\
\text { sujet }\end{array}$ & $\begin{array}{l}\text { Car- } \\
\text { bone } \\
\text { ingéré }\end{array}$ & $\begin{array}{l}\text { Car- } \\
\text { bone } \\
\text { fécal }\end{array}$ & $\begin{array}{l}\text { Car- } \\
\text { bone } \\
\text { urinaire }\end{array}$ & $\begin{array}{c}\text { Car- } \\
\text { bone } \\
\text { du } \\
\text { lait }\end{array}$ & $\begin{array}{c}\text { Car- } \\
\text { bone } \\
\text { du } \\
\mathrm{CH}_{4}\end{array}$ & $\begin{array}{c}\text { Car- } \\
\text { bone } \\
\mathrm{du} \\
\mathrm{CO}_{2}\end{array}$ & $\begin{array}{c}\text { Carbone } \\
\text { du } \\
\text { bilan }\end{array}$ & $\begin{array}{c}\text { Carbone } \\
\text { des } \\
\text { mat. } \\
\text { grasses }\end{array}$ & $\begin{array}{c}\text { Mat. } \\
\text { gr. } \\
\text { du } \\
\text { bilan }\end{array}$ & $\begin{array}{l}\text { Énergie } \\
\text { corres- } \\
\text { pondante }\end{array}$ \\
\hline & $\mathrm{g}$ & $g$ & $\mathrm{~g}$ & $g$ & $\mathrm{~g}$ & $\mathrm{~g}$ & $\mathrm{~g}$ & $\mathrm{~g}$ & $\mathrm{~g}$ & cal. \\
\hline & 946 & 32 & 55 & I66 & 32 & 396 & -27 & $-\overline{247}$ & 3 & -207 \\
\hline & 734 & $27 \mathrm{I}$ & 47 & 94 & 7 & 3 II & & $\begin{array}{r}2,9 \\
-\quad 1\end{array}$ & - & $-\quad 35$ \\
\hline
\end{tabular}


IV. - Comparaison des résultats obtenus par les deux modes de calcul :

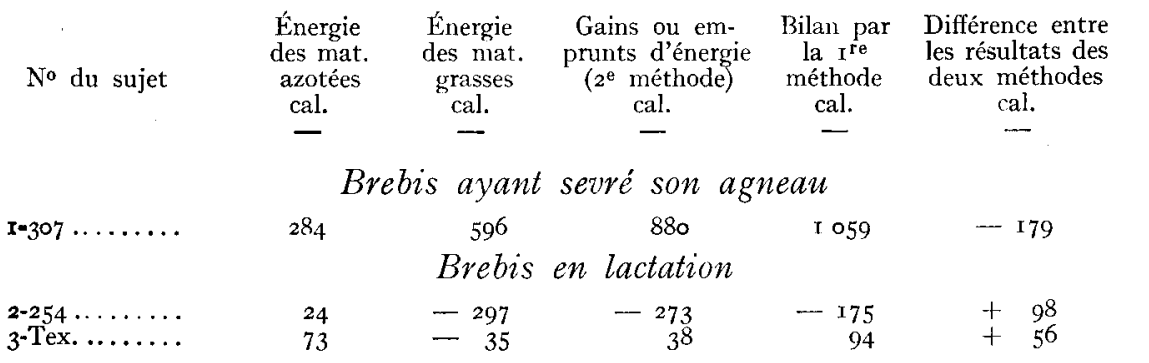

\section{IV. - DETERMINATION DE LACTION DYNAMIQUE SPECIFIQUE DES ALIMENTS INGERES EN FONCTION DE LEUR TENEUR EN MATIERE SECHE}

Nous avons montré, dans un travail précédent, que si l'on retire de l'énergie métabolisable correspondant à l'ingestion d'une ration donnée la somme de l'énergie d'entretien et de l'énergie de la production $\left(\mathrm{F}_{+}+\right.$ P), qui représente l'énergie nette, le résultat obtenu est très voisin de la quantité de matière sèche ingérée multipliée par un coefficient voisin de l'unité. Nous savons, en effet, que la consommation des aliments entraîne, par le jeu d'une série de phénomènes encore mal connus, une augmentation considérable de la consommation d'oxygène pendant et après le repas.

Avec les données précédentes, il nous est possible de vérifier 1'application de cette règle. Voici quels sont les résultats de ces opérations (tableau VII). Dans ce tableaı, 1'énergie métabolisable est représentée par M, E représente l'énergie d'entretien, déduite du poids vif de l'animal, et $\mathrm{P}$ représente l'énergie des tissus formés ou empruntés à l'organisme augmentée s'il y a lieu de l'énergie du lait. Enfin, la matière sèche est désignée par Ms.

TABLEAU VII

\begin{tabular}{|c|c|c|c|c|c|c|c|c|}
\hline & & & & Agneau & & & & \\
\hline & $\begin{array}{c}\text { Poids } \\
\text { du }\end{array}$ & $\begin{array}{c}\text { Energie } \\
\text { métabo- } \\
\text { lisable }\end{array}$ & $\begin{array}{l}\text { Energie } \\
\text { de } \\
\text { l'entretien }\end{array}$ & $\begin{array}{l}\text { Energie } \\
\text { du croît } \\
\text { ou du lait }\end{array}$ & $\begin{array}{c}\text { Energie } \\
\text { nette }\end{array}$ & $\begin{array}{l}\text { Matière } \\
\text { sèche }\end{array}$ & & $\frac{M-(\mathrm{E}+\mathrm{P})}{\mathrm{M}}$ \\
\hline $\begin{array}{l}\text { No du } \\
\text { sujet }\end{array}$ & sujet & $\mathrm{M}$ & & & $E+P$ & Ms & $\mathrm{y}-\mathrm{E}+\mathrm{P}^{\prime}$ & \\
\hline sujet & kg & cal. & cal. & cal. & cal. & $g$ & cal. & cal. parg \\
\hline$I=307 \ldots$ & - & 一 & - & $\longrightarrow$ & - & - & - & - \\
\hline$I-307 \ldots$ & I 8,3 & I 953 & 746 & 573 & I 319 & 576,8 & 634 & I,IO \\
\hline $2-314 \ldots$ & 19,2 & 2399 & 766 & 1178 & I 944 & 525,4 & 455 & 0,87 \\
\hline $3-3 \mathrm{I} 4 \ldots$ & 22,6 & I 859 & 840 & $5^{2} 5$ & I 365 & 546,2 & 494 & 0,90 \\
\hline $4-314 \ldots$ & 25,0 & I 555 & 888 & 210 & 1098 & $4 \circ 6,7$ & 457 & I,I 2 \\
\hline $5-3$ I $4 \ldots$ & $28, I$ & 2545 & 948 & 636 & I 584 & 836,0 & $96 \mathbf{T}$ & I 15 \\
\hline $6-314 \ldots$ & 30,7 & $189 \mathrm{I}$ & 997 & 70 & 1067 & 728,0 & 824 & I, I 3 \\
\hline $7-314 \ldots$ & $3 \mathrm{I}, \mathrm{O}$ & 2030 & I 003 & 525 & I 528 & 696,0 & 502 & 0,72 \\
\hline $8-314 \ldots$ & 32,8 & $24 \mathrm{I} 2$ & I 034 & 520 & I 554 & 81 7,0 & 858 & 1,05 \\
\hline & & & & Brebis & & & & . \\
\hline I-307 $\ldots$ & 69,2 & 4065 & I 430 & I 059 & 2489 & I 544 & I 576 & $\mathrm{I}, \mathrm{O} 2$ \\
\hline $2-254 \ldots$ & 64,2 & 5226 & 1371 & I 713 & 3084 & $2 \times 34$ & 2142 & $\mathrm{I}, \mathrm{OI}$ \\
\hline 3-Тех... & 53,8 & 4055 & 1252 & I 58 & 2410 & I 702 & I 645 & 0,97 \\
\hline
\end{tabular}


I1 est intéressant de comparer la moyenne de ces résultats, qui est exactement de $\mathrm{r}, 00 \pm 0,027$, à celle des mesures analogues que nous avons obtenues au cours de nos études sur le métabolisme des agneaux ne recevant que du lait.

La moyenne de ces dernières étant de I,06 $\pm 0,019$, il est aisé de voir que sa faible différence $-0,06 \pm 0,035$ avec le résultat que nous venons d'obtenir est dépourvue de signification. Nous pouvons donc admettre que l'énergie perdue au cours de la consommation desaliments et de leur digestion, qui s'ajoute à la dépense d'entretien, est égale approximativement à autant de calories qu'il y a de grammes dans la matière sèche ingérée.

\section{V. - CONCLUSION - UTILISATION DES RESULTATS OBTENUS AU CALCUL DES RATIONS DESTINEES AUX MOUTONS}

I1 est possible de tirer de ces constatations qui, comme nous le démontrerons dans un prochain mémoire, demeurent également valables pour les autres espèces, une méthode simple, précise et rapide pour le calcul des besoins alimentaires des moutons.

Nous savons que, chez les ruminants adultes, la quantité d'énergie métabolisable correspondant à une ration donnée, dont la composition chimique et la digestibilité sont connues, s'obtient par le calcul suivant : Énergie métabolisable $=$ (natière organique digestible $+1,25 \times$ matière grasse digestible) $\times 3,65$. Pour les jeunes animaux non sevrés ou très récenment sevrés, le coefficient 3,65 doit être remplacé par $4, \mathrm{I}$.

L'équation qui indique qu'un animal donnant des produits se tronve en équilibre énergétique s'écrit de la manière suivante :

Énergie métabolisable $=\mathrm{I}+\mathrm{P}+\mathrm{Ms} \times \mathrm{I}$ calorie, dans laquelle $F$, est l'énergie d'entretien, $P$, la ration de croissance ou de production laitière, et Ms, la quantité de matière sèche de la ration.

Voici un exemple simple destiné à montrer comment on peut utiliser cette équation, pour résoudre un problème de rationnement que nous avons choisi à dessein aussi près que possible des réalités pratiques : supposons que nous voulons nourrir des brebis en lactation pesant $60 \mathrm{~kg}$ donnant I $200 \mathrm{~g}$ de lait par jour, représentant I 500 calories, avec I $\mathrm{kg}$ de foin de luzerne et $5 \mathrm{~kg}$ de betteraves. Nous avons, pour compléter cette alimentation, l'intention d'utiliser un mélange composé d'une partie d'orge, une partie d'avoine et une partie de tourteau de lin. Nous désirons connaître quelle quantité de ce mélange concentré sera nécessaire pour équilibrer les besoins énergétiques de nos animaux.

I es données à utiliser pour les calculs sont les suivantes : 


\section{TABLEAU VIII}

\begin{tabular}{|c|c|c|c|c|}
\hline Aliment & $\begin{array}{c}\text { Mat. } \\
\text { organique } \\
\text { digestible } \\
0 / 00\end{array}$ & $\begin{array}{l}\text { Mat. } \\
\text { grasse } \\
\text { digestible } \\
\text { o/o0 }_{/ 00}\end{array}$ & $\begin{array}{l}\text { Energie } \\
\text { métabo- } \\
\text { lisable } \\
\text { o } \\
\text { cal. }\end{array}$ & $\begin{array}{c}\text { Matière } \\
\text { sèche } \\
\text { of } \\
\text { oo }\end{array}$ \\
\hline- & - & - & - & - \\
\hline Foin de luzerne, ordinaire & $46 \mathrm{I}$ & 14 & I 723 & 895 \\
\hline Betteraves fourragères $\ldots \ldots \ldots \ldots \ldots \ldots \ldots \ldots$ & 94 & 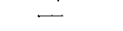 & $33^{8}$ & 120 \\
\hline 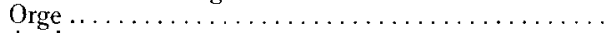 & 726 & 23 & 2718 & 872 \\
\hline$\ldots \ldots \ldots$ & 607 & 42 & 2367 & 888 \\
\hline Tourteau de lin extraction.. & 660 & 24 & 2484 & 894 \\
\hline
\end{tabular}

La partie de la ration formée de foin et de betteraves fournira aux animaux I723 $+338 \times 5=3$ 4I3 calories d'énergie métabolisable, pour une quantité de matière sèche de I 495 grammes.

Le mélange par parties égales d'orge, d'avoine et de tourteau de lin contient, par kg, 2523 calories d'énergie métabolisable et 885 grammes de matière sèche.

Si nous désignons par $x$ la quantité de mélange concentré que nous recherchons, l'équation exprimant l'équilibre des besoins énergétiques s'écrit de la manière suivante :

$$
34 \mathrm{I} 3+2523 x=\text { I } 325 \text { + I } 500 \text { + I } 495 \text { + } 885 x \text {, }
$$

la valeur $\mathrm{F}$ du besoin d'entretien d'une brebis de $60 \mathrm{~kg}$ étant de I 325 calories.

I a résolution de cette équation donne pour valeur de $x 553$ grammes. Tous les problèmes d'alimentation concernant les moutons peuvent se résoudre de cette manière, avec l'aide des tables faisant connaître la composition des aliments et les coefficients de digestibilité de leurs principaux éléments organiques. Il suffit de se rappeler la progression des besoins d'entretien en fonction du poids, qui sont les suivants :

\section{TABLEAU IX}

Besoins d'entretien des agneaux et brebis, en fonction de leur poids :

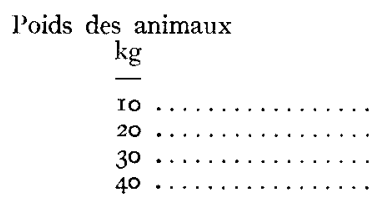

$$
\begin{gathered}
\mathrm{E} \\
\mathrm{cal} . \\
\hline 540 \\
780 \\
960 \\
\text { I } 090
\end{gathered}
$$

Poids des animaux

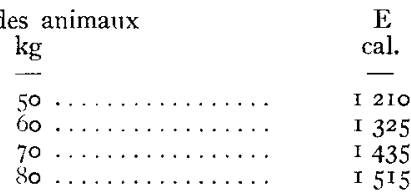

\section{RESUME}

I $\mathrm{Au}$ cours d'expériences de métabolisme avec l'aide des méthodes des échanges gazeux et des bilans azote-carbone, l'analyse des échanges énergétiques d'agneaux non sevrés et sevrés, de brebis en lactation et tarie, a été effectuée.

$2^{\circ}$ Les résultats obtenus prouvent que l'énergie supplémentaire 
entraînée par la consommation des aliments — qui, s'ajoutant à l'énergie proprement dite d'entretien, permet de connaître les pertes totales éprouvées par l'animal au cours du jeu de ses métabolismes, - est proportionnelle à la quantité de la matière sèche consommée.

$3^{\circ}$ La quantité de chaleur supplémentaire provenant de cette action dynamique spécifique des aliments est, pour l'espèce ovine, sensiblement égale à I calorie par gramme.

$4^{\circ}$ La conséquence de cet état de choses est la possibilité d'en déduire une méthode simple et suffisamment précise de calcul des rations destinées aux moutons.

\section{BIBLIOGRAPHIE,}

(I) L kiRoy (A. M.). - Rapports d'expériences faites sur l'alimentation du bétail. Publication du Conseil supérieur de l'Élevage, Paris I936.

(2) Liroy (A. M.). -- L'utilisation de l'Energie des aliments par les animaux. Bulletin Européen de la F.A.O., $\mathrm{N}^{\circ} 4$, fév. mars I 948 .

(3) LisRoy (A. M.). - Normes pour l'alimentation énergétique - Comptes rendus du Ve Congrès Intern. de Zootechnie, Paris I 949 .

(4) LEROY (A. M.), I,ERY (G.), ZEL'ś (S.). - Contribution à l'étude de l'utilisation du lait par les jeunes animaux. Annales de l'I.N.R. A., Zootechnie, $\mathrm{n}^{0} \mathrm{I}, \mathrm{I} 952,6 \mathrm{I}-77$.

(5) I, inoy (A. M.). - A propos du rassasiement de la vache laitière. Annales de l'I.N.R.A., Zootechnie, $\mathrm{n}^{\circ}$ 2, I952, 47-59.

(6) Ciarlis-Litry (C.), LERoy (A. M.), ZELTER (S.). - Élevage artificiel de l'agneau précocement sevré. Annales de l'I.N.R.A. Zootechnie, no 3 , I954, I69-187.

(7) LERoy (A. M.). - Tables de composition des aliments - L'F́levage rationnel - Librairie Hachette, Paris. 